\title{
RECOMMENDATION OF JD 19 ON NUTATION
}

\section{Recommendation 1}

The XXIId General Assembly of the International Astronomical Union, recognizing

1. that there are requirements to relate the J2000-celestial ephemeris pole (CEP) as defined by the 1980 IAU Theory of Nutation to the International Earth Rotation Service (IERS) CEP at J2000,

2. that the IERS provides accurate estimates of the offsets between these two poles,

3. that Very Long Baseline Interferometry (VLBI) and Lunar Laser Ranging (LLR) observations used by the IERS provide the most accurate data;

and considering

1. that a long series of observational data is required to separate longperiod nutations in longitude from precession, and long-period nutations in obliquity from obliquity rate, and to provide an improved estimate of precession, but

2. that there is an urgent need for an improved nutation numerical series for practical purposes;

urges

that observations of the offset of the celestial pole with respect to the pole defined by the 1980 IAU Theory of Nutation be made with the most precise techniques available including laser ranging to the Moon and very long baseline interferometry;

and asks

the International Earth Rotation Service to provide an empirical model for corrections to the 1980 IAU Theory of Nutation to be used for a priori estimates of the celestial ephemeris pole offsets. 
250

\section{Recommendation 2}

The XXIId General Assembly of the International Astronomical Union, recognizing

1. that an accepted geophysical nutation theory for the non-rigid Earth with oceans and atmosphere, including all known effects at the one tenth milliarcsecond level, is not yet available and requires further study,

2. that the Earth's nutation observations provide useful information about the physics of the Earth's interior,

establishes

an Inter-Commission Working Group on the Non-rigid Earth Nutation Theory to be organized by the Presidents of Commissions 4, 7, 19, under the leadership of V. Dehant, and

invites

the International Union of Geodesy and Geophysics (IUGG) to join the International Astronomical Union (IAU) in sponsoring this Working Group in order to form an IAU/IUGG Joint Working Group on this topic. 